\title{
NOUVELle
}

\section{Génétique de la schizophrénie}

Le complément, facteur de risque?

Dominique Campion ${ }^{1,2}$
${ }^{1}$ Centre hospitalier du Rouvray,

4 , rue Paul Eluard,

76301 Sotteville-lès-Rouen, France ;

${ }^{2}$ Inserm U1079, Génétique médicale

et fonctionnelle du cancer et des maladies neuropsychia-

triques, 76183 Rouen, France.

dominique.campion@univ-rouen.fr
> Durant des années, les études castémoins réalisées dans le but d'identifier des facteurs de risque génétique dans la schizophrénie, à partir de "gènes candidats », ont conduit à des résultats non reproductibles du fait de l'absence de rigueur statistique, notamment de la non prise en compte des tests multiples réalisés. En 2014, le Psychiatric genomics consortium (PGC) constitué par plusieurs dizaines d'équipes internationales a réuni une population d'environ 35000 patients et 45000 témoins à partir desquels a été menée une étude d'association à l'échelle, du génome (genome-wide association study, GWAS) [1]. Ce type d'étude explore, sans hypothèse a priori, les associations relevées avec des variants fréquents (en pratique, avec une fréquence de l'allèle mineur supérieure à $5 \%$ ) répartis sur l'ensemble des chromosomes. Compte tenu de l'existence de blocs haplotypiques, où ces variants sont en déséquilibre de liaison, on considère que la plupart des régions chromosomiques où sont présents des variants fréquents montrant une association avec la maladie, peuvent être détectées avec une bonne fiabilité par ces approches en génotypant quelques centaines de milliers de variants seulement. $\varepsilon n$ outre, les résultats peuvent être efficacement corrigés pour les tests multiples effectués avec, en corollaire, la nécessité de disposer de tailles d'échantillon très importantes pour parvenir à franchir le seuil de significativité genome-wide. Dans le cas présent, 108 locus associés à la schizophrénie ont été mis en évidence. Identifier une région avec un signal d'association positif n'équivaut toutefois pas à identifier un gène responsable. Dans 10 cas seulement, ce signal a pu être attri- bué à un variant non synonyme exonique (intéressant un exon). Dans les autres cas le signal était localisé hors des régions codantes, parfois à grande distance de gènes connus, suggérant que les variants détectés par GWAS affectent plus volontiers la régulation de l'expression génique plutôt que la structure protéique. Pour rendre les choses plus complexes encore, plusieurs signaux étaient souvent présents au sein d'une même région, rendant impossible de décider lequel sous-tendait l'effet biologique.

Pour commencer à avancer sur ces questions, Saker et ses collaborateurs [2] ont concentré leurs efforts sur la région donnant le signal d'association le plus fort, une région de 3,6 mégabases située sur le chromosome 6 et correspondant au complexe majeur d'histocompatibilité (CMH). Ils ont montré qu'au sein de cette région contenant notamment les gènes HLA (human leukocyte antigen) hautement polymorphes, l'essentiel du signal était concentré autour des 2 gènes, $C 4 A$ et $C 4 B$, codant le facteur 4 du complément. Cette microrégion est elle-même fort complexe puisque $\mathrm{C} 4 \mathrm{~A}$ et $\mathrm{C} 4 \mathrm{~B}$ varient en nombre de copies et existent en version courte ou longue, en raison de la présence, ou non, d'une insertion rétrovirale (HERV, human endogenous retroviruses) dans leur partie non codante. Cette insertion modifie l'expression du gène. Cependant, Sekar et ses collaborateurs sont parvenus à démêler cet écheveau en reliant ces caractéristiques structurales à des niveaux d'expression des gènes. Ils ont ensuite montré que différents niveaux de risque pour la schizophrénie étaient liés à différentes combinaisons entre le nombre de copies de C4 et le statut HERV : globalement, plus le niveau prédit d'expression de C4A est haut, plus le risque croît.

Une explication physiopathologique?

C4 est un constituant de la cascade du complément qui permet au système immunitaire inné de reconnaître débris cellulaires et pathogènes. Il est exprimé, en particulier, dans les astrocytes et les neurones. Du fait de sa localisation dans les synapses, il a été suggéré qu'il joue, au cours du développement, un rôle physiologique dans le processus d'élagage des synapses permettant l'élimination de celles sous-utilisées. Pour tester cette hypothèse, Sekar et ses collaborateurs ont étudié un modèle de souris déficiente pour C4. II faut noter à ce propos, que si chez l'homme, 2 gènes, $C 4 A$ et $C 4 B$, sont responsables de l'activation du facteur C3, permettant à ce dernier de se lier à ses cibles et de promouvoir leur phagocytose, un seul gène $C 4$ existe chez les rongeurs. Avec cette limitation, Sekar et ses collaborateurs ont pu montrer que les souris déficientes pour C4 ont un élagage synaptique réduit durant la maturation de leurs circuits neuronaux. À l'inverse, le facteur C4 est régulé positivement durant les périodes d'élagage. Ils spéculent alors que chez les patients, l'expression augmentée de C4A conduirait à un excès néfaste d'élagage.

Les auteurs relient ces faits aux multiples travaux ayant documenté, chez des patients schizophrènes, un amincissement anormal du cortex associatif ${ }^{1}$

${ }^{1}$ Le cortex associatif est un ensemble de régions cérébrales qui interviennent dans le traitement des informations complexes. 
sans qu'il n'y ait de perte cellulaire, ainsi qu'une diminution du nombre de synapses sur les neurones pyramidaux. Ils soulignent que, chez l'homme, la période cruciale pour l'élagage se situe durant l'adolescence et chez l'adulte jeune, ce qui corrèle avec l'âge de survenue de la schizophrénie. Évidemment, tout ceci demande confirmation mais impulsera sans nul doute des recherches visant à mieux comprendre cet aspect de la maturation neuronale. $\diamond$

Genetics of schizophrenia:

is the complement component 4 a risk factor?

\section{LIENS D'INTÉRÊT}

L'auteur déclare n'avoir aucun lien d'intérêt concernant les données publiées dans cet article.

\section{NOUVELLE}

\section{Le rôle critique de \\ la PI3-kinase dans la traversée des barrières de l'hôte par Listeria monocytogenes}

Grégoire Gessain $^{1-3}$, Olivier Disson ${ }^{1,2}$, Marc Lecuit ${ }^{1,2,4,5}$
$>$ Listeria monocytogenes $(\mathrm{Lm})$ est responsable de la listériose humaine, une infection systémique d'origine alimentaire dont le taux de mortalité atteint $30 \%$. Suite à l'ingestion d'aliments contaminés, $L m$ traverse l'épithélium intestinal, dissémine par voie sanguine, et atteint le système nerveux central et l'unité fœto-placentaire. $L m$ a donc la capacité de traverser les barrières intestinale, hémato-encéphalique et placentaire. Elle est à l'origine d'encéphalites et de méningites, ainsi que d'avortements et d'infections néonatales.

Mécanismes d'invasion cellulaire : rôle d'In|A et d'In|B

$L m$ est une bactérie intracellulaire facultative $^{l}$, qui est internalisée par les cellules non phagocytaires. Cette invasion est consécutive à l'interaction de deux protéines de surface bactérienne avec leurs récepteurs respectifs à la surface des cellules de l'hôte. Ces deux protéines bactériennes de surface sont l'internaline ( $\ln \mid A)$, qui interagit avec la $\varepsilon$-cadhérine ( $\varepsilon$ cad), une protéine

${ }^{1}$ Bactérie pouvant se multiplier au sein ou en dehors d'une cellule eucaryote.

permettant la formation des jonctions adhérentes entre cellules épithéliales, et $|n| B$, qui interagit avec $c-$ Met, le récepteur du facteur de croissance des hépatocytes (HGF) [1-4] $(\rightarrow)$.

L'interaction InIA-

Ecad est associée à

des réarrangements du cytosquelette d'actine nécessaires à l'entrée de

$(\rightarrow)$ Voir les Nouvelles de M. Lecuit et P. Cossart, $m / s n^{\circ} 12$, décembre 2001, page 1333, et $\mathrm{m} / \mathrm{s} \mathrm{n}^{\circ} 1$, janvier 2005, page 17 la bactérie dans la cellule. Cette interaction est spécifique d'espèce : la Ecad humaine est reconnue par $\ln \mid A$, alors que la Ecad de souris ne l'est pas [5]. Pour les espèces chez lesquelles l'interaction InIA-Ecad est possible, telles que le cobaye, la gerbille, l'homme et les souris humanisées (qui expriment la Ecad humaine ou une Ecad murine humanisée par la substitution $\varepsilon 16 \mathrm{P}^{2}$ ), InIA permet la traversée de l'épithélium intestinal $[6,7]$. L'interaction InIB-c-Met mime la voie de signalisation cellulaire de I'HGF : elle active la PI3-K (phosphoinositide 3-kinase), qui est nécessaire à l'internalisation de $\operatorname{Lm}$ [8].

${ }^{2}$ Substitution de l'acide glutamique $(\varepsilon)$ en position 16 par une proline $(P)$.

\section{RÉFÉRENCES}

1. Schizophrenia working group of the psychiatric genomics consortium. Biological insights from 108 schizophrenia-associated genetic loci. Nature 2014 ; $511: 421-7$.

2. Sekar A, Bialas AR, de Rivera $H$, et al. Schizophrenia risk from complex variation of complement component 4. Nature 2016 ; 530 : 177-83.

${ }^{1}$ Institut Pasteur, unité biologie des infections, 28, rue du Docteur Roux, F-75015 Paris, France ;

${ }^{2}$ Inserm, U1117, F-75015 Paris, France ;

3 Université Paris Diderot, Sorbonne Paris Cité, cellule Pasteur, F-75013 Paris, France;

${ }^{4}$ Institut Pasteur, centre national de référence et centre collaborateur de l'OMS Listeria, 28, rue du Docteur Roux, F-75015 Paris, France ;

${ }^{5}$ Université Paris Descartes, Sorbonne Paris Cité, service de maladies infectieuses et tropicales, hôpital universitaire

Necker-Enfants Malades, institut Imagine, F-75015 Paris, France.

olivier.disson@pasteur.fr marc.lecuit@pasteur.fr

Lm traverse les barrières intestinale et placentaire

InIA et InIB ont des contributions différentes lors du franchissement par $L m$ des barrières intestinale et placentaire. InIA est nécessaire et suffisante pour la traversée de l'épithélium intestinal. Elle interagit avec la Ecad située sur la face apicale des cellules caliciformes (CC) sécrétrices de mucus et accessible à leur face luminale (Figure IA) [9]. In|B, elle, n'est pas impliquée dans la traversée de la barrière intestinale [10]. En revanche, la traversée de la barrière placentaire requiert l'action conjointe et interdépendante d'InIA et d'InIB (Figure 1A) [7, 11].

Nous avons étudié les bases moléculaires et cellulaires des rôles respectifs d'InIA et d'InIB dans la traversée par $L m$ des barrières intestinale et placentaire [12]. Notre hypothèse de travail était la suivante : l'activité de la PI3-K étant nécessaire à I'internalisation de $L m$, cette enzyme 